\title{
Study of Effective Dentists Communication with Laboratory Technicians in Removable Partial Prosthesis
}

\author{
${ }^{1 *}$ Cheikh, Y., ${ }^{2}$ Badre, B., ${ }^{1}$ El. Assraoui, Kh., ${ }^{3}$ Karram, M.A., ${ }^{3}$ Khechane, Y., ${ }^{1}$ KAOUN, Kh. \\ ${ }^{1}$ Professor Department of Removable Prosthodontics \\ ${ }^{2}$ Professor, Department of Statistics, ${ }^{3}$ Dentist, Private Practice. \\ Faculty of Dentistry, Hassan II University of Casablanca, \\ Post code 21100, Casablanca, Morocco
}

\begin{abstract}
The aim of this study was to assess the means of communication used between the dentist and the dental technician in the removable partial prosthesis and to identify the difficulties encountered by the practitioners.

Materials and methods: a descriptive study has conducted among a sample of 289 dentists in Casablanca, Morocco. The survey is a questionnaire of 18 questions created by the researchers for the needs of the present study. The exclusion criteria comprised retirees, replacements, pedodontists, and exclusive orthodontists. Dentists were informed about the objectives of the survey, and their oral consent was obtained. Throughout the study, anonymity and respect for the identity of the data have been ensured.

Results: The results of this study show inadequate communication between dentists and laboratory technicians, modern means of communication are still less used compared to the traditional methods like written prescriptions (98,1\%) or phone (63,6\%). The use of the CAD/CAM technology was estimated at (16,4\%), in which the minority use it in RPDs (4,7\%). Communication difficulties can lead the dentist to end the relationship with the technician (63\%). Relationship difficulties are related to insufficient communication due to incomplete or poorly written instructions by the practitioner.

Conclusion: The quality of communication between dentist and laboratory technician is an essential component for the prosthetic project success. the training of dentists in communication with the dental technician and the use of new technologies is essential for good communication and better efficiency.
\end{abstract}

Key Words: Communication, Dental Technician, Dentist, Removable partial denture, Digital Dentistry.

\section{INTRODUCTION}

Removable partial dentures (RPDs) are custom medical devices made to restore the manducatory functions [1]. These complex treatments require clinical and laboratory steps, involving the dentist and the laboratory technician. It is a team effort that requires close collaboration and excellent communication between the different parties at each stage of construction of dental prostheses. Accurate information, skill, honesty and mutual respect are the basis of successful communication, and highest quality results [2]. However, in case of a design or construction prosthesis faults, the dentist is responsible for the laboratory technician's work [3]. According to the Medical Devices Directive (Directive $93 / 42$ EEC 1997), the dentist must provide adequate written documentation and instructions for the construction of a prosthesis, and the dental technician should fabricate the prosthesis by these instructions $[4,5,6]$.

Different means of communication are possible. Therefore, the dentist and the laboratory technician must know, master, and operate them correctly to optimize the design and prosthetic manufacturing.

In recent years, digital technology has invaded our daily lives and revolutionized dental practice. As a result, new technologies have modernized the manufacturing processes of dental prostheses and other means of communication between the practitioner and the laboratory technician, more reliable and faster, have appeared in parallel with these changes (digital photography, email, 
video conferencing, phone, etc.) [7,8]. However, most practitioners are not aware of the contribution of these innovations. In RPDs, the literature identified a lack of communication between the dentist and the laboratory technician [5,9-14]. In Morocco, no investigation has been carried out on this subject.

This work aims to describe the means of communication used between the practitioner and the dental technician in the removable metallic partial prosthesis, to identify the difficulties encountered by the practitioners, assess the place of the various digital processes in improving this communication and the quality of the prostheses produced.

\section{MATERIALS AND METHODS}

This descriptive study was conducted from December 10th, 2018 to February 9th, 2019. It targeted dentists in the liberal sector of Anfa Prefecture in the city of Casablanca. We chose the prefecture of Anfa, given the largest number of private practices equipped with new dental technologies in this area. The sample was randomly selected, and the study population for this report includes 289 practitioners from a total of 735 dentists. The survey was based on an 18-question questionnaire and used data collected in the literature. All the dentists were invited to participate in a face-to-face interview. The exclusion criteria comprised retirees, replacements, pedodontists, and exclusive orthodontists. Dentists were informed about the objectives of the survey in advance, and their oral consent was obtained. Throughout the study, anonymity and respect for the identity of the data were ensured. The questionnaire written in French was administered to practitioners by two investigators. Data from the questionnaire's responses were processed statistically using SPSS 10.0 software.

\section{RESULTS}

A total of 214 dentists took part in this study, representing a participation rate of (74.04\%). The sample's average in clinical practice time is 11.7 years, with a minimum of 0.33 years ( 4 months) and a maximum of 36 years (Table 1).

Table 1 : Vocational practice.

\begin{tabular}{lll}
\hline Variable & $\mathbf{n = 2 1 4}$ & $(\boldsymbol{\%})$ \\
\hline Duration of clinical experience : & & \\
Less than 5 years & 74 & 34,6 \\
Between 6 and 15 years & 72 & 33,6 \\
Between 16 and 25 years & 50 & 23,4 \\
More than 26 years & 18 & 8,4 \\
\hline
\end{tabular}

The laboratory work authorization form, which is one of communication means used between the dentist and the dental technician, is used by almost all practitioners in our sample $210(98,1 \%)$.

The digital communication tools allow creating a more direct link between the dentists and their collaborators. Yet, the majority of practitioners $202(94,4 \%)$ believe that these tools will have a positive impact on the quality of communication between the dentists and the dental technician. In this study, the majority of practitioners used these tools to transfer data $176(82,2 \%)$ and report an error $158(73,8 \%)$ to the technician.

$50 \%$ of the dentists reported sending additional data, if the technician requests a supplement of the written instructions supplied on the work authorization, in the form of sharing information (written or verbal) and digital photography by phone (phone call, WhatsApp) $(48,1 \%)$, video conferencing $(5,6 \%)$, work authorization $(3,7 \%)$ or Dropbox $(2,8 \%)$, (Table 2$)$.

Table 2: The means of communication used.

\begin{tabular}{|c|c|c|c|c|c|}
\hline Variable & & & $n$ & $(\%)$ & \\
\hline Using & $\mathbf{a}$ & work & & authorization & form \\
\hline Yes & & & 210 & 98.1 & \\
\hline No & & & 4 & 1.9 & \\
\hline
\end{tabular}

Using a digital communication tools 
International Journal of Advances in Scientific Research and Engineering (ijasre), Vol 7 (1), January -2021

\begin{tabular}{lcc}
\hline Positive impact & 202 & 94,4 \\
Negative impact & 12 & 5,6 \\
In which situation? & 176 & 82,2 \\
Data transfer & 158 & 73,8 \\
Error reporting & 2 & 0,9 \\
Others (oversight) & & \\
Sending additional instructions & 107 & 50 \\
Yes & 107 & 50 \\
No & & \\
Communication means used: & 103 & 48,1 \\
Phone (call, WhatsApp) & 12 & 5,6 \\
Videoconference & 6 & 2,8 \\
Drop box & 8 & 3,7 \\
Work authorization form & & \\
\hline
\end{tabular}

During diagnosis and treatment planning: only 58 (27\%) of practitioners claim to have an articulator in their dental office. They use it to mount diagnosis casts, analyse the occlusion, communicate and explain the treatment plan to the dental technician. The results showed that a small percentage of practitioners $6(2.7 \%)$ have a dental surveyor, of which only $4(1.8 \%)$ use it to determine the insertion path on diagnosis casts and produces design diagram of RPDs before sending them to the laboratory.

In the study population, 46 (21.5\%) of practitioners reported planning study casts and diagnosis of wax-up. They justify their choice for various reasons: Evaluate the anticipated final aesthetic, adjust the initial plan and improve communication with the technician and the patient (Table 3).

Table 3 : Use of Prosthodontic diagnosis tools in communication.

Variable

Possession of an articulator

Yes

No

Using the articulator: (if yes)

Mounting the casts

18,2

Analysing the occlusion

Improving communication

Possession of a dental surveyor:

Yes

No

Using a dental surveyor: (if yes)

Yes

No $n$

(\%)
39

44

36

$58 \quad 27$

20

16,8

41,9

2 


$\begin{array}{lcc}\text { Diagnosis wax-up: } & & \\ \text { Yes } & 46 & 21,5 \\ \text { No } & 168 & 78,5 \\ \text { Rationales: (if yes) } & & \\ \text { Control the occlusion } & 14 & 6,5 \\ \text { Anticipate final aesthetic } & 20 & 9,3 \\ \text { Improve communication } & 14 & 6,5\end{array}$

During prosthetic construction: 212 (97.2\%) of the dentists in our sample use conventional impressions. However, only 6 (2.8\%) of them have invested and also use the optical impression in RPDs. In this study, $82(38.3 \%)$ of practitioners claim to make themselves the design diagram of RPD's, however, most of them $132(61,7 \%)$ delegate it to a

technician.

Our study has demonstrated that more than half of dentists 123 (57,5\%) inform the prosthetist about the type of technique and materials required for the construction of prostheses.

Concerning the contribution of modern technologies, the majority of practitioners $176(82.2 \%)$ believe that the digitalization of RPDs manufacturing can improve the modalities of communication with the prosthetist. On the other hand, only 94 (43.9\%) of practitioners know Computer-aided Design Computer-aided Manufacturing (CAD/CAM) in dentistry and only 35 (16.4\%) of them use it in their practice. Among these practitioners, a minority (4.7\%) use it in RPD.

Practitioners who do not use the CAD/CAM justify this by their lack of interest in digital technology, the high cost of equipment, a few technicians trained and equipped to practice CAD/CAM, and for some, a preference for conventional techniques that give them satisfaction.

Traceability, in other countries, is supervised by an inspection body. However, in Morocco, it is not mandatory. Yet, more than half of the practitioners reported using a mean of traceability through documentation (Table 4).

Table 4 : Communication during removable partial denture construction.

Variable $n$

\section{Type of impression}

Conventional

212

97,2

Conventional and Optical

$6 \quad 2,8$

\section{RPD's design done by dentist:}

Yes

No

Specify type of material and techniques:

Yes

No

91

\section{Knowledge of CAD/CAM:}

Yes

94

43,9

No

120

56,1

Impact of using CAD/CAM on communication?

Useful 


\begin{tabular}{lrr} 
The use of CAD/CAM in dental prosthesis & & \\
Yes & 35 & 16,4 \\
No & 179 & 83,6 \\
In RPD & 10 & 4.7 \\
\hline
\end{tabular}

Obstacles: (if no)

Lack of interest $46 \quad 21,5$

Cost of equipment $129 \quad 60,3$

Lack of equipped laboratory

89 41,6

Satisfied with conventional techniques

4 1,9

\section{Traceability :}

Yes

No

90

42.1

Concerning the relationship between the dentist and the dental technician, $159(74.3 \%)$ of dentists said that their collaborators meet the deadlines, and only $55(25.7 \%)$ reported that technicians lack punctuality.

The types of errors made by technicians, reported by dentists in our sample were related to casting defects, aesthetics, errors in the framework design, occlusion and finishing defects. In this situation, $102(47.7 \%)$ of dentists requested meeting technician in a dental office, and the majority $150(70.1 \%)$ report resending the work back to the laboratory, and communicate the new instructions by work authorization, phone or email.

Difficult communication between the dentist and the laboratory can end this relationship. Our study has revealed that more than half of the dentists $136(63.6 \%)$ have already changed collaborators due to lack of skills and ongoing training, or the failure to meet deadlines (Table 5).

Table 5: Relationship difficulties with technician.

\section{Variable}

Respect of deadlines?

Yes

No

\section{Types of errors reported:}

inaccuracies in frameworks

Aesthetic

Errors in RPDs design

Finishing

Occlusion

Means of communication of defects:

Phone

Email

Laboratory work authorization form

Displacement of a technician $n$

(\%)
74,3 25,7

$55 \quad 25,7$

62

58

22

10,3

14

6,5

20

9,3 


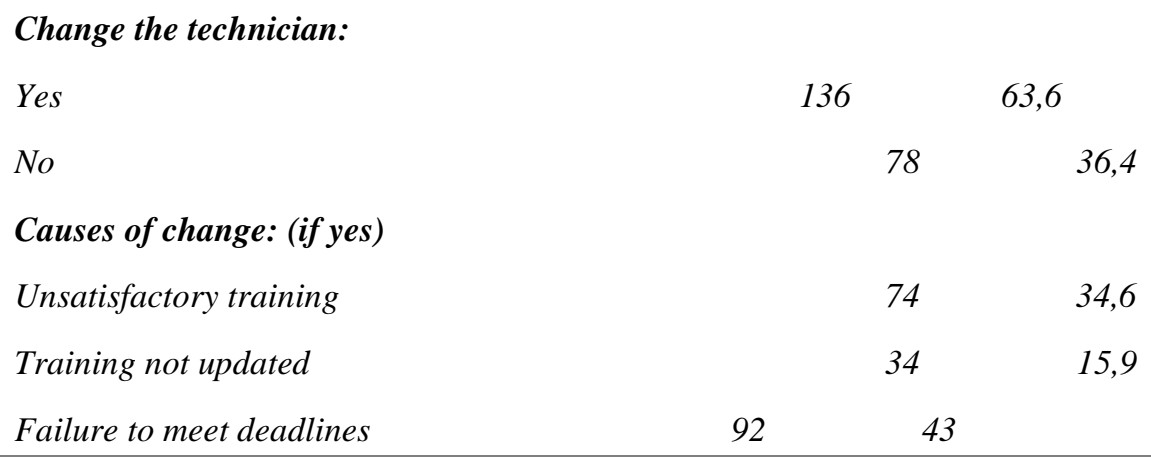

\section{DISCUSSION}

Our study was based on a sample of 289 practitioners with a response rate of $(74.04 \%)$. The studies conducted on this subject were based on smaller sample size $[15,16]$, and most often done with laboratory technicians $[5,9,13]$.

The results of this study have shown that the practitioner's clinical experience may influence the practitioner's communication with the laboratory technician, but not significantly. In contrast with this, Juszczyk et al. reported that there is a little difference between newly qualified_dentists and established dentists [17].

In the survey, almost all practitioners in our sample (98.13\%) has confirmed that the use of the work authorization form is the principal mean of communication. It agrees with a previous study conducted in Riyadh (97.6\%) [13]. This paper-based communication is insufficient and has several main drawbacks such as miscommunication between technicians and dentists and lack of visual interactivity [12].

The use of digital communication tools was more profitable for the dentists, whose average operational experience was approaching 11.7 years. In this respect, the majority of practitioners surveyed who know CAD/CAM and interested in its use were young people. The same result obtained in a study carried out in the United Kingdom [18].

Digital communication allows dentists and technicians the opportunity to make decisions quickly and concisely before, during, and after treatment [2]. (94.4\%) of practitioners, in this sample, are convinced of the crucial of digital communication tools in improving communication with the laboratory technician.

Nearly half $(48,1 \%)$ of respondents use digital photography to supplement the written instruction provided, which they transmit via different online sharing platforms (Drop Box, WhatsApp, Email). Sharing patient photos will avoid the need to meet the prosthetist in the office. These results approximate those of Berry et al. (63\%) [19].

The articulator and the dental surveyor are indispensable tools in the diagnosis, design and manufacture RPDs. In our study, few dentists use these tools in their practice; This implies that the articulator and dental surveyor may be considered as missing instruments in the communication between dentists and technicians. Similarly, the number of practitioners planning study casts and using the diagnosis wax-up, which is essential before making treatment decisions in RPD, was insufficient (21.5\%). The same results have been obtained by Lynch et al. were only (9\%) of dentists conducted the surveyed preliminary casts in RPD [9], and Haj-Ali et al. [20].

The essential step in prosthetic restoration is the impression. The optical impression enables more precise and instantaneous communication with dental technicians by transferring the data scanned to the laboratory, through a web portal. However, this procedure focussed only on small gap extent [21]. The results of our survey showed that the use of conventional impression techniques was by far the majority (97.1\%), with only (2.7\%) of practitioners associating the two techniques in RPD.

According to a recent study, $(84.2 \%)$ of laboratory technicians believe that the prosthesis design is the responsibility of the dentist only, but it is not provided in (54.5\%) of cases [22]. In the present study, (38.5\%) of practitioners reported that they draw and transmit the design of the RPD framework to the technician, this percentage is relatively similar to the one found by Haj-Ali et al. [20], but remains well below to what found in other studies $[10,13,16]$. It can be explained by trust between the practitioner and the dental technician. However, some studies deduced that this is mainly the result of a lack of competence of the practitioner $[6,10]$.

More than half of the respondents in our sample declared that they specify the type of material and the production procedure for the RPDs. This rate is well below than the results of previously published surveys of dental laboratories [16,23,24], but higher than that found by ASLAM et al. in India, which approximated (32\%) [25]. Therefore, the technician decides alone on the type of 
material or technique to use in the absence of instructions (alloy, artificial teeth). This communication gap can harm the condition of oral structures and on the patient's health [25].

On the other hand, our study has indicated that $16.4 \%$ of practitioners use CAD/CAM in their practice. This rate is significantly lower than that found in a previous study (41.8\%) [18]. The majority (60.3\%) of the practitioners of this study stated that the high investment initial cost was the most common barrier to the use of CAD/CAM in their practice. The second justification was the lack of dental laboratory equipped. The third justification was the absence of interest which seems to relate to the lack of continuing education and knowledge in computer science and technological innovation in dentistry. The enormous contribution of CAD/CAM technology in communication with the dental technician is undeniable. Our result has shown that $82.2 \%$ of practitioners are convinced of the fact that digitalization of dentistry is a mean to optimize communication. Practitioners in the UK share the same point of view (89.4\%) [18]. According to the same study, (13.8\%) of CAD/CAM users chose it to improve the relationship with the laboratory technician.

The main consequence of poor communication is the presence of defects in the work received from the laboratory. Results found that the most frequent errors are those related to inaccuracies in frameworks and aesthetic. It may be due to the insufficiency and inadequacies of the means of communication. Thus, the combination of photographs or videos of the patient's smile can be additional information for the aesthetic success of the prosthesis.

The most commonly used means of communication for reporting the error remains the phone for (63.6\%) of dentists, followed by asking the technician to come to the office (47.7\%), and sending instructions in work authorization (43.9\%). It can be concluded that the use of digital communication tools remains limited. These are preferably used by dentists to report an error to the technician and or to supplement clinical data, except in a study carried out in the UK, where digital means are most used, for example, email represents (76\%), and digital photography (63\%) [19].

Half of the practitioners stated that they were contacted by the technician to supplement the information previously provided, this can be due to inadequate means of communication, and the lack of competence of the dentist to submit all necessary information to the prosthetist. It proves the presence of failures in communication between the dentist and the technician. The findings were similar to the study of Aslam et al. (58\%) of prosthetists had to consult the dentist for clarifications [25]. It can be explained by the lack of sufficient basic training and clinical experience in managing the relationship with the prosthetist. Indeed, a study among dental students [16], concluded that the integration of training in communication with the laboratory technician in dental studies may increase its effectiveness and therefore reduce defects in the prosthetic part.

Despite ethical obligations that require practitioners to retain their patients' clinical data, only (57.9\%) of our sample use a mean of traceability. Juszczyk et al. concluded that the use of written documents in communication was preferred to verbal communication because it offers a permanent, signed and traceable record of a dentist's prescription [17]. Traceability is one of the motivations of the investment in the CAD/CAM, it allows to archive all the information in the patient's medical file (design of the framework, tooth reference, etc.). This information is essential during prosthetic maintenance.

\section{CONCLUSION}

Actually, with all available digital tools easy and accessible, communication between dentists and technicians must not be problematic. Unfortunately, our study showed inadequacies related to the lack of training in communicating with the technician and updating their knowledge in technological innovation in dentistry.

The main mean of communication must be unique, digital or paper format, then supplemented by other medical data via digital means for full traceability. By exploiting modern technologies in dentistry, the dentist can improve productivity and quality, communication with the dental technician.

Conflicts of Interest: The authors declare that there is no conflict of interest.

\section{Source of support : Nil}

\section{REFERENCES}

[1]. A. Griffin., ''Quality Assurance in Custom Dental Devices : A Technologist's Perspective ', Prim Dent J, 2017, vol. 6, no. 4, pp.41-43.

[2]. TR. Schoenbaum, YY. Chang. 'Dentist-technician collaboration in the digital age : enhancing outcomes through photography, teamwork, and technology'. J Calif Dent Assoc, 2011, vol. 39, no. 8, pp.559-567.

[3]. M. Dumont, “'Assurance responsabilité professionnelle’’. Actual Odontostomatol (Paris), 2002, n. 218, pp. 173-183. 
[4]. EC Medical Devices Directive No 10. Guidelines to Medical Devices Directive 93/42/EEC for manufacture of custom made dental devices., 1997, Dublin : Department of Health and Children.

[5]. G.P. Kilfeather, C. D. Lynch, A. J. Sloan, et al., "Quality of communication and master impressions for the fabrication of cobalt chromium removable partial dentures in general dental practice in England, Ireland and Wales in 2009', J Oral Rehabil., 2010, vol. 37, no.4, pp.300-5.

[6]. L. Sui, Wu. Xueying, Wu. Shuhong, et al., 'Quality of Written Instructions for Dental Prostheses in China', Journal of Prosthodontics, , 2014, vol 23, no.8, pp. 602-9.

[7]. F. Mangano, J. A. Shibli, and T. Fortin, 'Digital Dentistry: New Materials and Techniques', International Journal of Dentistry, Vol. 2016, Article ID 5261247, 2 pages.

[8]. I. Fouilloux, Hurtado, M.Begin., 'Prothèse composite : la communication clinicien prothésiste'”. Stratégie prothétique, 2002, $\mathrm{n}^{\circ} 2$.

[9]. C. D. Lynch, P. F. Allen, Quality of written prescriptions and master impressions for fixed and removable prosthodontics : a comparative study. Br Dent J, 2005, vol.198, no.1, pp.17-20.

[10]. A. Radhi, C. D. Lynch, A. Hannigan, 'Quality of written communication and master impressions for fabrication of removable partial prostheses in the Kingdom of Bahrain', J. of Oral Reha., 2007, vol. 34, pp. 153-157.

[11]. S. H. Leeper, ' 'Dentist and laboratory: A 'love-hate' relationship.'’ Dent Clin North Am, 1979, no.23, pp. 87-99.

[12]. I. F. Alshiddi, 'Communication between Dental Office and Dental Laboratory: From Paper-Based To Web-Based'. PODJ, 2014, vol.34, no.3, pp.555-559.

[13]. H. M. Al-alsheikh, "Quality of communication between dentists and dental technicians for fixed and removable prosthodontics King Saud University’’ J. of Dent. Sci. , 2012, vol.3, pp.55-60.

\{14]. A. Farias-Neto, R. S. G. da Silva, A. da Cunha Diniz, 'Ethics in the provision of removable partial dentures', Braz. J. Oral Sci, 2012, vol.11, no.1.

[15]. M. Gueye, N. Thioune, EL. Didia, et al., "Collaboration entre le cabinet dentaire et le laboratoire de prothèse : enquête auprès des chirurgiens-dentistes de Dakar', Rev Col Odonto-Stomatol Afr Chir Maxillo-fac, 2014, vol. 21, no.4, pp. 19-23.

[16]. GR. Parry, JL. Evans, A. Cameron, "Communicating Prosthetic Prescriptions from Dental Students to the Dental Laboratory: Is the Message Getting Through?’ J Dent Educ., 2014, vol.78, no.12, pp.1636-42.

[17]. A. S. Juszczyk, R. K. F. Clark, D. R. Radford, “UK dental laboratory technicians' views on the efficacy and teaching of clinical-laboratory communication”, British Dental Journal, 2009, vol. 206, no.10.

[18]. D. Tran, M. Nesbit, H. Petridis, "Survey of UK dentists regarding the use of CAD/CAM technology”, Bri. Br. Dent. J., 2016, vol. 221, no.10, pp. 639-644.

[19]. J. Berry, M. Nesbit, S. Saberi and H. Petridis, "Communication methods and production techniques in fixed prosthesis fabrication : a UK based survey.'” Part 1 : Communication, Bri. Dent. J., 2014, vol.217, no.6, pp.643 E12.

[20]. R. Haj-Ali, F. Al quran, O. Adel, "Dental laboratory communication regarding removable dental prosthesis design in the UAE'. J Prosthodont., 2012, vol. 21, no. 5, pp. 425-8.

[21]. C. Batisse, G. Bonnet, P. Bongert, et al. '’Optical Impression and Removable Partial Denture : An Accurate and Actual Solution?', J Dent Oral Health, 2017, vol.3, no. 6, pp. 78.

[22]. S. Arbab ali, N. Khalifa, M. N. Alhajj, “Communication Between Dentists and Dental Technicians During the Fabrication of Removable Partial Dentures in Khartoum State, Sudan’, Acta Stomatol Croat., 2018, vol.52, no.3, pp. $246-253$.

[23]. H. Tulbah, E. Alhamdan, A. Alqahtani et al., "Quality of communication between dentists and dental laboratory technicians for fixed prosthodontics in Riyadh, Saudi Arabia', The Saudi Dent. J.,2017, vol. 29, pp.111-116.

[24]. C. Stewart., 'An audit of dental prescriptions between clinics and dental laboratories'. Br Dent J. 2011, vol.211, no.3, E5.

[25]. A. Aslam, B. Ahmed, A. Ali Azad, et al., "Bridging the gap. Quality of communication between prosthodontists and lab technicians", Pak Oral Dental J, 2015, vol. 35, no.1, pp.148-51. 